\title{
A CHARACTERIZATION OF THE UNIFORM CONVERGENCE POINTS SET OF SOME CONVERGENT SEQUENCE OF FUNCTIONS
}

\author{
OLENA KARLOVA ${ }^{1,2}$
}

\begin{abstract}
AвSTRACT. We characterize the uniform convergence points set of a pointwisely convergent sequence of real-valued functions defined on a perfectly normal space. We prove that if $X$ is a perfectly normal space which can be covered by a disjoint sequence of dense subsets and $A \subseteq X$, then $A$ is the set of points of the uniform convergence for some convergent sequence $\left(f_{n}\right)_{n \in \omega}$ of functions $f_{n}: X \rightarrow \mathbb{R}$ if and only if $A$ is $G_{\delta}$-set which contains all isolated points of $X$. This result generalizes a theorem of Ján Borsík published in 2019.
\end{abstract}

\section{Dedicated to the memory of Ján Borsik}

\section{INTRODUCTION}

Let $X$ be a topological space, $(Y,|\cdot-\cdot|)$ be a metric space; $B(a, r)$ and $B[a, r]$ be an open and a closed ball in $Y$ with a center $a \in Y$ and a radius $r>0$, respectively. By $\partial A$ we denote a boundary of a set $A$.

Let $\mathscr{F}=\left(f_{n}\right)_{n \in \omega}$ be a sequence of functions $f_{n}: X \rightarrow Y$. We denote $P C(\mathscr{F})$ the set of all points $x \in X$ such that the sequence $\left(f_{n}(x)\right)_{n \in \omega}$ is convergent in $Y$. Therefore, we define the limit function $f(x)$ by the rule $f(x)=\lim _{n \rightarrow \infty} f_{n}(x)$ for all $x \in P C(\mathscr{F})$. Let us observe that the set $P C(\mathscr{F})$ can be represented in the form

$$
P C(\mathscr{F})=\bigcap_{k \in \omega} \bigcap_{n \in \omega} f_{n+\omega}^{-1}\left(B\left[f(x), \frac{1}{k+1}\right]\right) .
$$

If every function $f_{n}$ is continuous, then the set $P C(\mathscr{F})$ is $F_{\sigma \delta}$ in $X$. Hans Hahn [6] and Wacław Sierpiński [11] proved independently that the converse proposition is true for metrizable $X$ and $Y=\mathbb{R}$, that is, for every $F_{\sigma \delta}$-subset $A$ of a metrizable space $X$ there exists a sequence $\mathscr{F}$ of real-valued continuous functions $f_{n}: X \rightarrow \mathbb{R}$ such that $A=P C(\mathscr{F})$.

After appearance of this theorem many results were obtained in similar directions: other types of convergence and other classes of functions were considered (see, for instance, [1, 4, 7, 8, 9, 10, 12, 13, 14]). Ján Borsík studied in [1], in particular, the uniform convergence points set of a (convergent pointwisely) sequence of functions.

Definition 1. A sequence $\left(f_{n}\right)_{n \in \omega}$ of functions $f_{n}: X \rightarrow Y$ between a topological space $X$ and a metric space $(Y,|\cdot-\cdot|)$ is uniformly Cauchy at a point $x_{0} \in X$, if for every $\varepsilon>0$ there exist a neighborhood $U$ of $x_{0}$ and a number $n_{0} \in \omega$ such that $\left|f_{n}(x)-f_{m}(x)\right|<\varepsilon$ for all $n, m \geq n_{0}$ and $x \in U$.

Let $U C(\mathscr{F})$ be a set of all points with the uniform Cauchy property for a sequence $\mathscr{F}=\left(f_{n}\right)_{n \in \omega}$. It is easy to see that if $\left(f_{n}\right)_{n \in \omega}$ is convergent pointwisely on $X$ to a function $f: X \rightarrow Y$, then

$$
U C(\mathscr{F})=\left\{x \in X: \forall \varepsilon>0 \exists U \ni x_{0} \exists n_{0} \forall n \geq n_{0}\left|f_{n}(y)-f(y)\right|<\varepsilon \forall y \in U\right\} .
$$

Moreover, in this case $U C(\mathscr{F})$ is the set of all points of the uniform convergence of $\mathscr{F}$. Borsík proved the following result.

2010 Mathematics Subject Classification. Primary 54C30, 26A21; Secondary 54C50.

Key words and phrases. set of points of uniform convergence; uniformly Cauchy sequence. 
Theorem A (Borsík, [1]). Let $X$ be a metric space and $A \subseteq X$. Then $A=U C(\mathscr{F})$ for some convergent sequence $\mathscr{F}=\left(f_{n}\right)_{n \in \omega}$ of functions $f_{n}: X \rightarrow \mathbb{R}$ if and only if $A$ is $G_{\delta}$ and contains all isolated points of $X$.

This short note is inspirited by the above mentioned paper of Ján Borsík. We generalize his theorem on a wider class of topological spaces.

Definition 2. A topological space $X$ is $\omega$-resolvable if there exists a partition $\left\{X_{n}: n \in \omega\right\}$ of $X$ by dense subsets.

For crowded spaces (i.e., spaces without isolated points) the class of all $\omega$-resolvable spaces includes all metrizable spaces, Hausdorff countably compact spaces, arcwise connected spaces, etc. [2]

The main result of our note is the following theorem.

Theorem 1. Let $X$ be a perfectly normal $\omega$-resolvable space and $A \subseteq X$. The following conditions are equivalent:

(i) A is a set of all points of uniform convergence for some convergent sequence $\left(f_{n}\right)_{n \in \omega}$ of functions $f_{n}: X \rightarrow \mathbb{R}$;

(ii) $A$ is a $G_{\delta}$-set which contains all isolated points of $X$.

\section{Proof of Theorem 1}

The implication $(i) \Rightarrow$ (ii) follows immediately from the equality (1.2).

(ii) $\Rightarrow$ (i). Let $\left(G_{n}\right)_{n \in \omega}$ be a sequence of open sets in $X$ such that $A=\bigcap_{n \in \omega} G_{n}, G_{n+1} \subseteq G_{n}$ for every $n \in \omega$ and let $G_{0}=X$.

Since $X$ is perfectly normal, for every $n \in \mathbb{N}$ there exist continuous functions $\varphi_{n}, \psi_{n}: X \rightarrow[0,1]$ such that $\varphi_{n}^{-1}(0)=\overline{G_{n}}$ and $\psi_{n}^{-1}(0)=X \backslash G_{n}$. Then every function $\alpha_{n}: X \rightarrow[-1,1]$ defined by the formula $\alpha_{n}=\varphi_{n}-\psi_{n}$ has the following properties:

$$
\begin{array}{ll}
\alpha_{n}(x)<0 & \forall x \in G_{n}, \\
\alpha_{n}(x)=0 & \forall x \in \partial G_{n}, \\
\alpha_{n}(x)>0 & \forall x \in X \backslash \overline{G_{n}} .
\end{array}
$$

We consider functions $\beta_{n}: X \rightarrow[-1,1]$ defined by the rule

$$
\beta_{n}(x)=\max _{i \leq n} \alpha_{i}(x)
$$

for all $x \in X$. Then we claim that

$$
\partial G_{n}=\beta_{n}^{-1}(0)
$$

for every $n \in \mathbb{N}$. We need to prove $\beta_{n}(x)=0 \Leftrightarrow \alpha_{n}(x)=0$ for every $x \in X$. Assume that $\beta_{n}(x)=0$. Then $\alpha_{i}(x) \leq 0$ for all $i \leq n$ and $\alpha_{k}(x)=0$ for some $k \leq n$. Then $x \in \overline{G_{i}}$ for all $i \leq n$. If $x \notin \partial G_{n}$, then $x \in G_{n} \subseteq G_{i}$ for all $i \leq n$, consequently, $\alpha_{k}(x)<0$, a contradiction. Hence, $x \in \partial G_{n}$ and $\alpha_{n}(x)=0$. Conversely, if $\alpha_{n}(x)=0$, then $x \in \partial G_{n} \subseteq \overline{G_{n}} \subseteq \overline{G_{i}}$ for all $i \leq n$. In consequence, $\alpha_{i}(x) \leq 0$ for all $i<n$ and $\beta_{n}(x)=0$.

Now we put

$$
\gamma_{n}(x)=\min _{i \leq n}\left|\beta_{i}(x)\right|
$$

and notice that

$$
\gamma_{n}^{-1}(0)=\bigcup_{i \leq n} \partial G_{i}
$$


Finally, let

$$
\delta_{n}(x)= \begin{cases}0, & x \in \overline{G_{n}} \\ \gamma_{n}(x), & x \in X \backslash \overline{G_{n}} .\end{cases}
$$

Obviously, the functions $\beta_{n}, \gamma_{n}$ and $\delta_{n}$ are continuous and $\delta_{n} \leq \gamma_{n}$.

We put $U_{k, 0}=F_{k, 0}=\emptyset, k \in \mathbb{N}$. For all $k, n \in \mathbb{N}$ we define

$$
U_{k, n}=\gamma_{n}^{-1}\left(\left[0, \frac{1}{k}\right)\right) \quad \text { and } \quad F_{k, n}=\delta_{n}^{-1}\left(\left[\frac{1}{k}, 1\right]\right) .
$$

The sets $U_{k, n}$ and $F_{k, n}$ satisfy the following conditions:

(A) $U_{k, n}$ is open and $F_{k, n}$ is closed in $X$,

(B) $\overline{U_{k+1, n}} \subseteq U_{k, n} \subseteq U_{k, n+1}$,

(C) $F_{k, n} \subseteq F_{k+1, n} \cap F_{k, n+1}$,

(D) $\delta_{n}^{-1}((0,1])=\bigcup_{k \in \mathbb{N}} F_{k, n}=X \backslash\left(\bigcup_{i=1}^{n} \partial G_{i} \cup G_{n}\right)$,

(E) $\gamma_{n}^{-1}(0)=\bigcup_{i=1}^{n} \partial G_{i}=\bigcap_{k \in \mathbb{N}} U_{k, n}$,

(F) $U_{k, n} \cap F_{k, n}=\emptyset$

for all $k, n \in \mathbb{N}$.

Moreover, the sets $G_{n}$ satisfy the property

(G) $\left(\partial G_{n} \backslash \partial G_{n-1}\right) \cap \partial G_{i}=\emptyset, i<n, n \in \mathbb{N}$.

Since the most of properties are evident, we prove only $(C)$ and $(G)$.

$(C)$. It is enough to prove that $F_{k, n} \subseteq F_{k, n+1}$. Fix $x \in F_{k, n}$ for some $k, n \in \mathbb{N}$. Then $\delta_{n}(x) \geq \frac{1}{k}$ and, in consequence, $x \notin \overline{G_{n}}$. Therefore, $\alpha_{n}(x)>0$ and $\alpha_{n+1}(x)>0$. Hence, $\beta_{n}(x)>0$ and $\beta_{n+1}(x)>0$. Since $\gamma_{n}(x)=\delta_{n}(x) \geq \frac{1}{k}$, the inequality $\left|\beta_{i}(x)\right| \geq \frac{1}{k}$ holds for all $i \leq n$. In particular, $\left|\beta_{n}(x)\right|=\beta_{n}(x) \geq \frac{1}{k}$. Then $\left|\beta_{n+1}(x)\right|=\beta_{n+1}(x) \geq \beta_{n}(x) \geq \frac{1}{k}$. Thus, $\delta_{n+1}(x)=\gamma_{n+1}(x)=$ $\min _{i \leq n+1}\left|\beta_{i}(x)\right| \geq \frac{1}{k}$ and $x \in F_{k, n+1}$.

$(G)$. Fix $x \in \partial G_{n} \backslash \partial G_{n-1}$. Since $x \in \partial G_{n}, x \in \overline{G_{n}} \backslash G_{n}$. Then $x \in \overline{G_{n-1}}$, because the sequence $\left(G_{n}\right)_{n \in \omega}$ is decreasing. Moreover, $x \notin \partial G_{n-1}$ and therefore $x \in G_{n-1}$. Again, since $\left(G_{n}\right)_{n \in \omega}$ decreases, $x \in G_{i}$ for all $i<n$. Hence, $x \notin \partial G_{i}, i<n$.

Since $X \backslash \bar{A}$ is an open subset of the $\omega$-resolvable space $X$, it is $\omega$-resolvable also. Hence, there exists a sequence $\left(B_{k}\right)_{k \in \omega}$ of mutually disjoint subsets of $X \backslash \bar{A}$ such that

$$
X \backslash \bar{A}=\bigcup_{k \in \omega} B_{k}
$$

and each set $B_{k}$ in dense in $X \backslash \bar{A}$.

For every $x \in X$ we put

$$
f(x)=\left\{\begin{array}{lc}
\frac{1}{n}, & x \in \partial G_{n} \backslash \partial G_{n-1} \text { for some } n \in \mathbb{N}, \\
0, & \text { otherwise. }
\end{array}\right.
$$

Notice that $f$ is correctly defined because of property $(G)$.

Now let

$$
C_{k, n}=\left(U_{k, n} \backslash U_{k, n-1}\right) \cup\left(B_{k} \cap\left(F_{k, n} \backslash F_{k, n-1}\right)\right)
$$


and

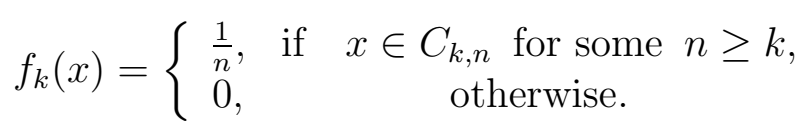

In order to show that $\left(f_{k}\right)_{k \in \omega}$ converges to $f$ pointwisely on $X$ we fix $x \in X$.

If $x \in \bigcup_{n \in \omega} \partial G_{n}$, then we put $N=\min \left\{n \in \omega: x \in \partial G_{n}\right\}$. Therefore, since $\partial G_{0}=\emptyset$, property (G) implies that $N \in \mathbb{N}$ and $x \in \partial G_{N} \backslash \bigcup_{i<N} \partial G_{i}=\partial G_{N} \backslash \partial G_{N-1}$. Hence, $f(x)=\frac{1}{N}$. Then by (F) we conclude that $x \in \partial G_{N} \subseteq \bigcap_{k \in \mathbb{N}} U_{k, N}$ and $x \notin \bigcap_{i<N-1} \partial G_{i}=\bigcap_{k \in \mathbb{N}} U_{k, N-1}$. In consequence, taking into account (B) we conclude that there exists $K \in \omega$ such that $x \in U_{k, N} \backslash U_{k, N-1}$ for all $k \geq K$. Therefore, $f_{k}(x)=\frac{1}{N}=f(x)$ for all $k \geq K$. Hence, $\lim _{k \rightarrow \infty} f_{k}(x)=f(x)$.

If $x \notin \bigcup_{n \in \omega} \partial G_{n}$, then $f(x)=0$. Let $\varepsilon>0$ and $n \in \mathbb{N}$ be such that $\frac{1}{n}<\varepsilon$. Using (F) we conclude that $x \notin \bigcup_{i<n} \partial G_{i}=\bigcap_{k \in \mathbb{N}} U_{k, n}$. Then, taking into account (B), we obtain that there is $K_{1} \in \mathbb{N}$ such that $x \notin U_{k, n}$ for all $k \geq K_{1}$. But $x \in \bigcup_{k \in \omega} B_{k}$ and the sets $B_{k}$ are disjoint, so there is $K>K_{1}$ such that $x \notin \bigcup_{k \geq K} B_{k}$. Therefore, we have $x \notin \bigcup_{k \geq K}\left(U_{k, n} \cup B_{k}\right)$.

Assume that $k \geq K$. Consider the case $x \in U_{k, k}$. Then we choose the minimal number $m \leq k$ such that $x \in U_{k, m}$. Then $m>n$ (indeed, if $m \leq n$, then $x \in U_{k, m} \subseteq U_{k, n}$, a contradiction). In particular, $m>1$ and $x \in U_{k, m} \backslash U_{k, m-1} \subseteq C_{k, m}$. Therefore, $f_{k}(x)=\frac{1}{m}<\frac{1}{n}<\varepsilon$. Now we consider the case $x \notin U_{k, k}$, then (B) implies that $x \notin U_{k, n}$ for any $n \leq k$. But $x \notin B_{k}$. Hence, $x \notin C_{k, n}$ for every $n \geq k$. Thus, $f_{k}(x)=0<\varepsilon$.

Now we prove that $A=U C(\mathscr{F})$ for $\mathscr{F}=\left(f_{n}\right)_{n \in \omega}$. Fix $x \in A, \varepsilon>0$ and let $n_{0} \in \mathbb{N}$ be such that $\frac{1}{n_{0}}<\varepsilon$. Since $x \in A$, we conclude that $x \in G_{i}$ for every $i$ and then $x \notin \bigcup_{i \leq n_{0}} \partial G_{i}=\bigcap_{k \in \mathbb{N}} U_{k, n_{0}}$. Then there exists $k_{0} \geq n_{0}$ with $x \notin U_{k_{0}-1, n_{0}}$. Therefore, property (B) implies that $x \notin \overline{U_{k, n_{0}}}$ for all $k \geq k_{0}$.

We consider an open neighborhood

$$
U=G_{n_{0}} \backslash \overline{U_{k_{0}, n_{0}}}
$$

of $x$ in $X$. Take an arbitrary $u \in U$ and $k \geq k_{0}$. Since $u \in G_{n_{0}}$, we have that $u \in G_{i}$ and then $u \notin \partial G_{i}$ for any $i \leq n_{0}$. Therefore,

$$
f(u) \in\left[0, \frac{1}{n_{0}}\right) .
$$

Let us observe that (B) and (C) imply that $C_{k, n} \subseteq U_{k, k} \cup\left(B_{k} \cap F_{k, k}\right)$ for all $k \geq n$. Therefore, if $u \notin U_{k, k} \cup\left(F_{k, k} \cap B_{k}\right)$, then $f_{k}(u)=f(u)=0$.

Let us consider the case $u \in U_{k, k}$. Then we take the minimal $i \leq k$ such that $x \in U_{k, i}$. Notice that $i>n_{0}$ (indeed, if $i \leq n_{0}$, then (B) implies that $u \in U_{k, i} \subseteq U_{k, n_{0}} \subseteq U_{k_{0}, n_{0}}$, a contradiction). In particular, $i>1$ and then $u \in U_{k, i} \backslash U_{k, i-1} \subseteq C_{k, i}$. Thus, $f_{k}(u)=\frac{1}{i}<\frac{1}{n_{0}}$.

In the case $u \in F_{k, k}$ we choose the minimal $j \leq k$ with $u \in F_{k, j}$. Observe that $j>n_{0}$ (indeed, if $j \leq n_{0}$, then $u \in F_{k, j} \subseteq F_{k, n_{0}}$, and so (D) implies $u \notin G_{n_{0}}$, which is impossible). In particular, $j>1$ and $u \in B_{k} \cap\left(F_{k, j} \backslash F_{k, j-1}\right) \subseteq C_{k, j}$. Therefore, $f_{k}(x)=\frac{1}{j}<\frac{1}{n_{0}}$.

Thus, we proved that in any case $f_{k}(u) \in\left[0, \frac{1}{n_{0}}\right)$. Hence,

$$
\left|f(u)-f_{k}(u)\right| \leq \frac{1}{n_{0}}<\varepsilon .
$$

Therefore, $A \subseteq U C(\mathscr{F})$.

Now we prove that $U C(\mathscr{F}) \subseteq A$. In order to do this we fix $x \notin A$ and show that $x \notin U C(\mathscr{F})$ in this case. Let $n_{0}=\max \left\{n \in \omega: x \in G_{n}\right\}, \varepsilon=\frac{1}{n_{0}+1}-\frac{1}{n_{0}+2}, U$ be an open neighborhood of $x$ and let $k_{0} \in \omega$. Notice that $x \in G_{n_{0}} \backslash G_{n_{0}+1}$.

Consider the case $x \in \overline{G_{n_{0}+1}}$. Then $x \in \overline{G_{n_{0}+1}} \backslash G_{n_{0}+1}=\partial G_{n_{0}+1}$. Therefore, (E) implies that $x \in \partial G_{n_{0}+1} \subseteq \bigcap_{k \in \mathbb{N}} U_{k, n_{0}+1}$. On the other hand, $x \in G_{n_{0}}$. So, $x \notin \partial G_{i}$ for any $i \leq n_{0}$. Using 
(E) we conclude that $x \notin \bigcup_{i \leq n_{0}} \partial G_{i}=\bigcap_{k \in \mathbb{N}} U_{k, n_{0}}$. Then, there exists $k_{1} \in \mathbb{N}$ such that $x \notin U_{k_{1}, n_{0}}$. Therefore, (B) implies that $x \notin \overline{U_{k, n_{0}}}$ for all $k \geq k_{1}$. Hence, there exists $k>\max \left\{k_{0}, n_{0}\right\}$ such that $x \in U_{k, n_{0}+1} \backslash \overline{U_{k, n_{0}}}$.

Since the set $\bigcup_{n \leq n_{0}+1} \partial G_{n}$ is nowhere dense in $X$, there is a nonempty open set $V$ such that $V \subseteq$ $U \cap\left(U_{k, n_{0}+1} \backslash \overline{U_{k, n_{0}}}\right) \backslash \bigcup_{n \leq n_{0}+1} \partial G_{n}$. Take $u \in V$. Then $f(u) \in\left[0, \frac{1}{n_{0}+2}\right]$, because $u \notin \bigcup_{n \leq n_{0}+1} \partial G_{n}$, and $f_{k}(u)=\frac{1}{n_{0}+1}$, since $u \in U_{k, n_{0}+1} \backslash U_{k, n_{0}}$. Therefore,

$$
\left|f(u)-f_{k}(u)\right| \geq \frac{1}{n_{0}+1}-\frac{1}{n_{0}+2}=\varepsilon .
$$

Now we assume that $x \notin \overline{G_{n_{0}+1}}$. Then $x \in G_{n_{0}} \notin \overline{G_{n_{0}+1}}$. Therefore, $x \notin \bigcup_{i \leq n_{0}+1} \partial G_{i}=\gamma_{n_{0}+1}^{-1}(0)$. Consequently, $\gamma_{n_{0}+1}(x)>0$. So, there exists $m_{0} \in \mathbb{N}$ such that $\frac{1}{m_{0}}<\gamma_{n_{0}+1}(x)$. But $x \notin \overline{G_{n_{0}+1}}$. Therefore, $\delta_{n_{0}+1}(x)=\gamma_{n_{0}+1}(x)>\frac{1}{m_{0}}$. Hence, $x \in \operatorname{int} F_{m_{0}, n_{0}+1}$.

By property $(\mathrm{C})$, there exists a number $k>\max \left\{k_{0}, m_{0}, n_{0}\right\}$ such that $x \in \operatorname{int} F_{k, n_{0}+1}$. Since $x \in G_{n_{0}}, x \notin F_{k, n_{0}}$. Then the set

$$
G=\left(U \backslash \overline{G_{n_{0}+1}}\right) \cap\left(\operatorname{int} F_{k, n_{0}+1} \backslash F_{k, n_{0}}\right)
$$

is an open neighborhood of $x$. Since $\bigcup_{n \leq n_{0}+1} \partial G_{n}$ is nowhere dense in $X$ and $B_{k}$ is dense in $X \backslash \bar{A}$, there exists a point $v \in X$ such that

$$
v \in\left(G \backslash \bigcup_{n \leq n_{0}+1} \partial G_{n}\right) \cap B_{k} .
$$

Then $f_{k}(v)=\frac{1}{n_{0}+1}$, since $v \in C_{k, n_{0}+1}$, and $f(v) \in\left[0, \frac{1}{n_{0}+2}\right]$, because $v \notin \bigcup_{n \leq n_{0}+1} \partial G_{n}$. Hence,

$$
\left|f(v)-f_{k}(v)\right| \geq \frac{1}{n_{0}+1}-\frac{1}{n_{0}+2}=\varepsilon .
$$

Therefore, $A=U C(\mathscr{F})$.

Remark 1. Actually, we use in the proof only the fact that the boundary of every open set in a topological space $X$ is a functionally closed set. It is find out that this is a characterization of perfectly normal spaces. Moreover, the following conditions are equivalent:

(i) $X$ is a perfectly normal space;

(ii) every closed nowhere dense subset of $X$ is functionally closed.

Evidently, (i) $\Rightarrow$ (ii). In order to prove (ii) $\Rightarrow$ (i) we take a closed set $F \subseteq X$. Since $\partial F=F \backslash \operatorname{int} F$ is closed and nowhere dense, there exists a continuous function $g: X \rightarrow[0,1]$ such that $\partial F=g^{-1}(0)$. Let us define $f: X \rightarrow[0,1]$ by $f(x)=g(x)$ if $x \in X \backslash \operatorname{int} F$ and $f(x)=0$ if $x \in \operatorname{int} F$. It is easy to see that $f$ is continuous and $F=f^{-1}(0)$. Therefore, $X$ is perfectly normal by Vedenisoff's theorem.

Remark 2. By one of reviewers, in Theorem 1 it is sufficient to assume that $Y$ is a non-discrete metric space.

Remark 3. Any topological vector space is $\omega$-resolvable [2]. So, the space of all continuous function $C_{p}([0,1])$ equipped with the topology of pointwise convergence is an example of perfectly normal $\omega$-resolvable space which is not metrizable.

Remark 4. Eric K. van Douwen proved in [3, Theorem 5.2] that there exists a crowded countable regular space which cannot be represented as a union of two disjoint dense subsets. It is easy to see that this space is perfectly normal and not $\omega$-resolvable.

\section{ACKNOWLEDGEMENT}

I am very grateful to the reviewers for their careful reading of the manuscript and valuable remarks which allowed to improve the paper. 


\section{REFERENCES}

[1] Borsik, J.: Points of uniform convergence and quasicontinuity, Eur. J. Math. (2019)

[2] Comfort, W.W., Garcia-Ferreira, S.: Resolvability: a selective survey and some new results, Topology Appl. 74 (1996), 149-167.

[3] van Douwen E.K. Applications of maximal topologies, Topology Appl. 51 (2) (1993), 125-139.

[4] Drahovský, Š., Šalát, T., Toma, V.: Points of uniform convergence and oscillation of sequences of functions , Real Anal. Exchange 20 (1994/95), 753-767.

[5] Engelking R.: General Topology. Revised and completed edition. Heldermann Verlag, Berlin (1989).

[6] Hahn, H.: Über die Menge der Konvergenzpunkte einer Funktionenfolge, Arch. d. Math. u. Phys. 28 (1919-1920), 34-45.

[7] Holá, L., Holý, D.: Pointwise convergence of quasicontinuous functions and Baire spaces, Rocky Mountain J. Math. 41 (2011), 1883-1894.

[8] Maslyuchenko, V. K., Mykhaylyuk, V. V., Sobchuk, O. V.: Inverse problems of the theory of separately continuous mappings, Translation from Ukrainian J. Math. 44 (9) (1992), 1209-1220.

[9] Natkaniec, T., Wesołowska, J.: On the convergence of $\omega_{1}$ sequences of real functions, Acta Math. Hung. 90 (2001), $335-350$.

[10] Natkaniec, T., Wesołowska, J.: Sets of ideal convergence of sequences of quasicontinuous functions, J. Math. Anal. Appl. 423 (2015), 924-939.

[11] Sierpiński, W.: Sur l'ensemble des points de convergence d'une suite de fonctions continues, Fund. Math. 2 (1921), 41-49.

[12] Wesołowska, J.: On set of convergence of convergence points of transfnite sequence of quasi-continuous functions, Tatra Mt. Math. Publ. 28 (2004), 21-27.

[13] Wesołowska, J.: On sets of convergence points of sequences of some real functions, Real Anal. Exchange $\mathbf{2 5}$ (1999-2000), 937-942.

[14] Wesołowska, J.: On sets of discrete convergence points of sequences of real functions, Real Anal. Exchange 29 (2003-2004), 107-120.

1 Jan Kochanowski University in Kielce, Poland; ${ }^{2}$ Chernivtsi National University, Ukraine E-mail address: maslenizza.ua@gmail.com 